\title{
Review
}

\section{Food, nutrition, physical activity and cancer prevention. Authoritative report from World Cancer Research Fund provides global update}

\author{
First published online 8 May 2008
}

From little acorns mighty oak trees can grow. On 1 November last year the World Cancer Research Fund (WCRF) released its second comprehensive report on the role of food, nutrition and physical activity in the prevention of cancer ${ }^{(1)}$. This imposing volume, the product of an exhaustive and meticulous 5-year review of the world's scientific literature by a pantheon of nutritional scientists, epidemiologists and biostatisticians, comes 10 years after the first WCRF report on the same topic. This new report also comes exactly a quarter of a century after the establishment of the WCRF progenitor, the American Institute for Cancer Research (AICR). That AICR 'acorn' first took root in the inner-suburban soil of east coast USA.

Before the 1980s the central narrative of cancer epidemiology focused on the role of specific chemical and physical agents, including occupational exposures and ambient environmental pollutants. The idea of chemical (and radiation) mutagenesis and carcinogenesis was pivotal to theories of cancer causation during the second and third quarters of the last century. From the mid-1970s, however, evidence of hormonal influences on cancer risk accrued. So too did evidence of dietary influences - from inter-population comparisons, from studies of migrants moving between dietary environments and from individual-level epidemiological studies.

The idea that human diets might influence cancer risk and that appropriate diets could reduce cancer risk opened up a new research vista. It offered new prospects for prevention. Further, here was an opportunity for individuals and families to take some control of their own risks of cancer. Regulations and enforcement agencies are needed to curb exposure to occupational, industrial and environmental carcinogens, but individuals, well informed, could change their food choices. The AICR set out to gather and disseminate that information.

The venture succeeded: the community responded and donated. In the early 1990s AICR went global, establishing the World Cancer Research Fund. That body, WCRF, then embarked on its ambitious first major report: Diet, Nutrition and the Prevention of Cancer: a Global Perspective. Meanwhile, after two decades of methodological toil, epidemiologists were realizing that diet-cancer causal relationships were complex, often elusive, and compounded by difficulties in measuring usual diets over time, estimating nutrient intakes and taking account of appropriate life stages and long cancer 'incubation' processes. That first report produced a spectrum of findings: some clear-cut, some guarded, some uncertain. It also pointed to the likely importance of dietary profiles-at-large, as composite mixed 'exposures', in cancer causation. Does the diet as a whole, by whatever biological path(s), exert greater influence than the sum of its ingredient parts?

That first WCRF report of 1997, manifestly authoritative, became influential at national and international levels. It provided a sound basis for public education and food policies to minimize cancer risks, and it influenced research directions.

This second, wider-ranging, report draws on a vast international body of published research, which the panel and its specialist side-teams methodically identified, evaluated and winnowed. Evidence of close interrelationships between dietary habits, physical activity, relative weight and cancer risks has firmed, as have insights into lifecourse' processes that influence cancer risk over time. That more longitudinal perspective has been applied to the role of early childhood nutrition and growth rates, relative weight and, in girls, the onset of puberty.

That same longitudinal perspective has led to some other new terrain. First, the report recommends exclusive breast-feeding for the first six months of life, citing evidence on cancer (and other diseases) that 'sustained, exclusive breastfeeding is protective for the mother as well as the child'. Second, the report makes a generic case for healthy dietary behaviours and professionally supervised nutritional support for cancer patients.

The twenty-one-person panel was chaired by the UK's Professor Michael Marmot. It was supported by a methodology task force, a mechanisms working group, and a globally diverse set of expert centres commissioned to do systematic reviews (UK, USA, Italy and Netherlands). The panel faced a demanding dual task - to agree on the strength of the evidence relating specific cancers to specific dietary components, and to formulate and communicate the resultant recommendations. 
The report comprises twelve substantial chapters arranged as Background, Evidence and Judgements, and Recommendations. The second part contains the panel's strength-of-evidence assessments. These are well displayed in a comprehensive colour-coded matrix that arrays eighteen cancer sites/types against several dozen foods and nutrients and indices of physical activity, anthropometry and infant feeding. The cells indicate, visually, whether that particular relationship (if studied) was assessed as convincing, probable or limited.

Each of the resultant recommendations (Part 3) is expressed as both a public health goal and a personal recommendation. The former, importantly, are for populations; the latter are for people: communities, families, individuals. The recommendation on meat consumption is illustrative: (i) population target, not more than $300 \mathrm{~g}$ red meat per capita per week; and (ii) personal target for meat-eaters, not more than $500 \mathrm{~g}$ red meat/week.

The report makes eight general recommendations relating to: body weight; physical activity; energy-dense foods and drinks; plant food intake; animal food intake; alcoholic drinks; foods that have been preserved or processed and food preparation methods; and dietary supplements.

Interestingly, the report evinces a growing recognition of the role of energy restriction in reducing disease risk and, hence, the desirability of achieving an energy balance at a level to which man, over many millennia, is evolved and adapted. A recent remarkable epidemiological study in Cuba provides further evidence of the health benefits of energy restriction ${ }^{(2)}$. An emerging research challenge, then, is to elucidate factors that cause fast early-life growth, accelerated sexual maturity and adult tallness in life (empirically, a strong correlate of risk of some cancers). Overnutrition is presumably a key factor.

There is more to come in subsequent volumes. The Report states that:

The panel is aware that, as with other diseases, the risk of cancer is also modified by social, cultural, economic and ecological factors. ... Identifying the deeper factors that affect cancer risk enables a wider range of policy recommendations and options to be identified. This is the subject of a separate report to be published in late 2008 .

Given growing awareness of the adverse environmental impacts of intensified food production around the world - including impacts on water supplies, soil fertility, ecological systems and greenhouse gas emissions - we can hope that this further report will seek a balance between sustaining the good health of people and that of their planetary life-support systems.

Professor A.J. McMichael National Centre for Epidemiology and Population Health The Australian National University Canberra, ACT 0200, Australia Email: Tony.McMichael@anu.edu.au

\section{References}

1. World Cancer Research Fund/American Institute for Cancer Research (2007) Food, Nutrition, Physical Activity and the Prevention of Cancer: a Global Perspective. Washington DC: AICR; available at http://www.dietandcancerreport.org

2. Franco M, Orduez P, Caballero B, Tapia Granados JA, Lazo M, Bernal J, Guallar E \& Cooper RS (2007) Impact of energy intake, physical activity, and population-wide weight loss on cardiovascular disease and diabetes mortality in Cuba, 1980-2005. Am J Epidemiol 166, 1374-1380. 